January 2000

\title{
The Malingering of Psychotic Disorders
}

Michael R. Harris M.D.

University of Louisville School of Medicine

Follow this and additional works at: https://jdc.jefferson.edu/jeffjpsychiatry

Part of the Psychiatry Commons

Let us know how access to this document benefits you

\section{Recommended Citation}

Harris, Michael R. M.D. (2000) "The Malingering of Psychotic Disorders," Jefferson Journal of Psychiatry. Vol. 15 : Iss. 1 , Article 7.

DOI: https://doi.org/10.29046/JJP.015.1.003

Available at: https://jdc.jefferson.edu/jeffjpsychiatry/vol15/iss1/7

This Article is brought to you for free and open access by the Jefferson Digital Commons. The Jefferson Digital Commons is a service of Thomas Jefferson University's Center for Teaching and Learning (CTL). The Commons is a showcase for Jefferson books and journals, peer-reviewed scholarly publications, unique historical collections from the University archives, and teaching tools. The Jefferson Digital Commons allows researchers and interested readers anywhere in the world to learn about and keep up to date with Jefferson scholarship. This article has been accepted for inclusion in Jefferson Journal of Psychiatry by an authorized administrator of the Jefferson Digital Commons. For more information, please contact: JeffersonDigitalCommons@jefferson.edu. 


\title{
The Malingering of Psychotic Disorders
}

\author{
Michael R. Harris, MD
}

\begin{abstract}
The phenomenon of malingered psychosis is examined through a review of the available literature. Possible motivations for malingering are discussed, and clinical indicators of feigned psychotic symptoms are reviewed. The methods discussed focus on the inpatient evaluation of suspected malingerers and include discussions of interview techniques and psychometric testing to supplement clinical impressions. A differential diagnosis is presented, and techniques for confronting a malingering patient are reviewed.
\end{abstract}

\section{INTRODUCTION}

Faking mental illness to avoid unpleasant tasks is an ancient human pasttime. Greek authors refer to the story of Odysseus, who pretended to be insane to avoid participation in the Trojan War. He hitched both an ox and a horse to his plow and began sowing salt into the ground instead of seeds, but was found out when he swerved the plow to avoid his infant son placed in its path. And the Bible tells of David who, afraid of the wrath of jealous king Achish, "altered his behavior in public and acted like a lunatic in front of them all, scrabbling on the double doors of the city gate and dribbling down his beard."

More recently, social and legal pressures have prompted some to seek the security of hospital wards and government entitlements by pretending to be mentally ill. In DSM-IV (1), these behaviors are distinguished diagnostically by their motivation. Feigned mental or physical illness whose purpose is primarily to take on the cared-for role of a patient is called factitious disorder, while the same presentation with the goal of avoiding work, military service, legal proceedings, or for obtaining money, is called malingering. It should be noted that, while factitious disorders are coded on Axis I in the DSM-IV diagnostic scheme, malingering is a "V code" — that is, a condition not attributable to a mental illness.

The reasons a patient may fake a mental illness are many, but psychiatrists in non-forensic settings are likely to see patients malingering for one of three reasons (2):

1. Seeking a psychiatric diagnosis to obtain financial gain from disability or workman's compensation;

2. Faking an illness to get prescription medications; or

3. Seeking admission to a psychiatric hospital for food, shelter, and/or protection from the legal system. 
This paper will focus on the phenomenon of feigned psychotic disorders, with an emphasis on inpatient evaluation of suspected malingerers. This focus was chosen for several reasons. First, "psychosis" is a term that covers a wide range of clinical presentations. This makes psychosis attractive to malingerers, since inconsistent symptoms may be seen as simply atypical. Second, most psychiatrists are very reluctant to diagnose malingering in an emergency setting due to the critical nature of the situation and the frequent lack of reliable collateral information. Malingerers determined to be admitted for psychiatric care are likely to succeed, and unless the inpatient team is alert to the possibility of malingering the patient will probably never be found out. And finally, most inpatient psychiatry units will have the professional staff and facilities to carry out the series of evaluations necessary to detect malingering. These may not be available or practical on an outpatient basis.

\section{TYPICAL GHARACTERISTICS OF PSYCHOTIC SYMPTOMS}

In DSM-IV, the term psychosis is restricted to delusions, prominent hallucinations, disordered speech, disordered behaviors, and catatonia (1). Disorders of speech, such as word salad, neologisms, or derailment, are difficult to imitate, and do not appear to be popular with malingerers. Similarly, bizarre behaviors tend to be tiring and are difficult to sustain for extended periods of time. Most research in malingered psychosis has concentrated on hallucinations, delusions, and catatonia, in an attempt to find characteristics that help divide true from malingered symptoms. In the following sections, we will review the literature on psychotic symptoms on the presumption that accurate knowledge of legitimate psychiatric symptoms will make the false symptoms of malingering easier to detect. As we shall see, this is not as easy as it sounds.

\section{HALLUCINATIONS}

Hallucinations are sensory perceptions that occur in the absence of external stimuli. They can involve any sensory modality, but auditory and visual hallucinations are the most commonly reported types. Hallucinations are not specific to any disorder and in fact may occur in anyone under certain conditions such as metabolic disturbances, drug ingestion, alcohol withdrawal, falling asleep, or waking up (3). Numerous psychiatric disorders may have associated hallucinations, including schizophrenia, depression, mania, and some personality disorders. To evaluate the possibility of malingered hallucinations, the clinician must consider the characteristics of hallucinations that have been reported in the literature.

Table 1 lists some common and uncommon characteristics of hallucinations, based on a review of the available literature. We must always remember that there are no "rules" about hallucinations, but there are common presentations that are internally consistent and consistent with known psychiatric conditions. Most hallucinations, of any type, are consistent with a patient's delusional system and appear to 
TABLE 1.

Common and Uncommon Characteristics of Hallucinations

\begin{tabular}{|c|c|c|}
\hline Characteristic & Common & Uncommon \\
\hline Frequency on a given day & Intermittent & Continuous \\
\hline Day to day frequency & Not every day & Daily \\
\hline Duration of episodes & 1 hour or less & More than 1 hour \\
\hline Circumstances & $\begin{array}{l}\text { Occur when others are } \\
\text { present }\end{array}$ & Occur only when alone \\
\hline Multiple Sensory Modes & Only one mode at a time & $\begin{array}{l}\text { Multiple modes } \\
\text { simultaneously }\end{array}$ \\
\hline $\begin{array}{l}\mathrm{Pt} \text { able to reduce intensity } \\
\text { by certain actions }\end{array}$ & Yes & No \\
\hline Origin of $\mathrm{AH}$ & Outside of head & Inside of head \\
\hline Gender of AH & Male and female & Only one gender \\
\hline Quality of AH & Clear, conversational & Vague, threatening \\
\hline Content of commands & Mundane, benign & Violent, specific instructions \\
\hline Response to commands & $\begin{array}{l}\text { Engage in internal } \\
\text { dialogue }\end{array}$ & $\begin{array}{l}\text { Commit harmful or illegal } \\
\text { acts "on command" }\end{array}$ \\
\hline Color of VH & Full color & Single color, black and white \\
\hline Size of VH & Normal size & Miniature, giant \\
\hline Quality of VH & Appear realistic & Bizarre, frightening \\
\hline VH coordinate with $\mathrm{AH}$ & No & Yes (e.g. talking figures) \\
\hline
\end{tabular}

serve an internal purpose-for example, a patient with unacceptable guilt may experience accusatory auditory hallucinations (4) or experience hallucinations in other modalities associated with their delusions such as olfactory sensations or visual hallucinations.

Verbal hallucinations usually speak in clear language that is easily understood. The majority of patients report that they hear both male and female voices at different times, and that the voices are often identified by the patient (e.g., family members, famous people, God). Relatively few patients experience continuous hallucinations; rather, the experiences occur intermittently for periods of less than one hour at a time, and in some studies did not even occur every day $(3,5,6)$. Although verbal hallucinations commonly occur in the presence of other people, some patients report that being alone makes the hallucinations more prominent (5). Most patients can localize the voices as coming from a source outside of their heads and can differentiate the hallucinated voices from the voices of real people. The majority of patients with chronic verbal hallucinations report that they have developed numerous strategies for improving their tolerance of this annoying situation. Reported coping mechanisms include seeking interpersonal contact, doing work or leisure activities, physical relaxation techniques, redirecting attention to other thoughts, and using antipsychotic medications (7). Overall, the literature indicates that verbal hallucinations tend to have a predictable nature that becomes integrated into the patient's life. Malingering patients will be unlikely to hold this view of "voices" and may "overplay" their symptoms, as discussed later. 
Command hallucinations, voices that suggest or direct a course of action to the patient, pose particular difficulty for the examiner. They are easy to malinger to provide a psychotic motive for otherwise illegal or destructive acts. In most studies, however, patients reported that their command hallucinations involved mundane, daily activities rather than sudden, atypical impulses $(3,8)$. Most persons with schizophrenia reported that they were usually able to ignore direct commands from their voices, and in the cases when they complied with the commands the patients noted that the voices gave them good advice. An interesting finding by Leudar and coworkers (8) was that voices would sometimes "nag" the patient about certain commands if they were not obeyed. Rather than repeating the same instructions, the voices will rephrase them, talk louder, or even start to curse the patient. Overall, it appears that a majority of verbal hallucinations carry on a kind of internal dialogue with the patient in which the patient is an active participant. Claims of irresistible, repetitious command hallucinations that offer a convenient excuse for malefaction should be explored in detail, since the potential secondary gain (release from legal responsibility) is quite high. It should also be noted, however, that command hallucinations with violent content increase the risk of suicide in acutely psychotic patients, so careful consideration of all factors is required before dismissing these hallucinations as unreliable.

In most studies acutely psychotic patients reported hallucinating in only one sensory modality at a time. Several authors $(3,5,6)$ report that while many psychotic patients reported hallucinations in modes other than auditory, they were described as uncommon and transitory. However, Chesterman (9) reminds us that multi-modal hallucinations are not always malingering and may be infrequently reported because clinicians do not probe for their presence. As an example, he cites a study by Rupert (10) in which the incidence of olfactory hallucinations in a schizophrenic sample rose from $5 \%$ to $83 \%$ when specific questions were asked. A subtle but important distinction is that while many patients may hallucinate in more than one sensory modality simultaneously, the hallucinations usually involve the same theme without being integrated. For example, the patient may experience persecutory voices combined with disturbing visions, but only rarely do visual hallucinations also speak to the patient as if the patient were watching a real person talk.

Visual hallucinations are almost always of normal-sized people in normal colors. Reports of bizarre apparitions, "little green men," or "animal friends" should arouse suspicion of malingering (11). Although visual hallucinations are common in schizophrenia and other psychotic disorders, they are generally infrequent compared to the incidence of auditory hallucinations $(3,5)$. Visions that talk back to the patient appear to be more characteristic of severe personality disorders or bereavement than of psychotic disorders. Like auditory hallucinations, visual hallucinations are usually complex and well-formed and are consistent with the patient's delusional system (4). Psychotic visual hallucinations will appear the same whether the patient's eyes are open or closed. This contrasts with hallucinations caused by substance abuse or withdrawal, which tend to become clearer and brighter when the eyes close (4). 


\section{DELUSIONS}

Delusions, fixed false beliefs not acceptable to a patient's culture and not alterable by reasoning, are common in psychotic patients. Although malingering patients may report the sudden onset of a delusion, in reality delusions tend to develop gradually over a period of weeks or months (12). When a delusional system exists, the patient's daily actions are usually in accord with their delusions for a period of time before presentation. If interviewing the patient and collecting collateral information indicates that a patient has been acting "normally" during the period when he claimed extreme paranoia or control by alien forces, the suspicion of malingering should increase. Most psychotic patients have reasonably complete and detailed delusions, so a patient who is unsure of details or answers "I don't know" to multiple questions about details must also be suspected of feigning or exaggerating their symptoms.

\section{CATATONIA AND MUTISM}

Catatonia (extreme psychomotor disturbance) and mutism (inability or unwillingness to speak) are more difficult to malinger and maintain for extended periods of time. True catatonia and mutism are seen in acute schizophrenic episodes, conversion disorder, and metabolic disturbances. The syndrome typically presents with generalized psychomotor retardation, posturing, negativism, automatic obedience, and waxy flexibility (1). Hopkins (13) has reported two cases of catatonia related to conversion disorder that required artificial ventilation. Catatonia may also present with extreme agitation, aggression, and excitement that goes on for extended periods and may result in hyperpyrexia and self-injurious behavior. Extended observation on the inpatient unit will usually demonstrate that the malingering patient can indeed move and talk, and that outbursts of aggression are volitional and can be interrupted by staff interventions.

\section{WHEN TO SUSPECT MALINGERING}

David Rosenhan's 1973 paper (14) describing his "plants" of nonpsychotic volunteers on inpatient psychiatry units seemed to indicate that psychiatrists didn't recognize malingering unless they specifically looked for it. When they did look for it, they saw it in some cases where it did not exist. In Witztum's report on malingering in the Israeli military (15), he noted that two dozen military conscripts were repeatedly diagnosed as malingering to avoid compulsory military service when in fact they were severely mentally ill. Part of the explanation for the misdiagnoses involved the customs and traditions of ultraorthodox Jews living in Israel, which were unfamiliar to the initial examiners. Yates and coworkers (16) polled psychiatric residents working in the emergency room and found that, although a substantial number of patients were at least suspected of malingering, none received "malingering" as their primary Axis I diagnosis and only $2.6 \%$ received it as a secondary diagnosis. These 
studies demonstrate the wide variation in the ability and willingness of psychiatrists to consider and investigate malingering.

Cunnien (17) has recommended a threshold model for consideration of malingering (Table 2). Although malingering may in theory be added to any differential diagnosis, for practical purposes it need only be considered in the context of an inconsistent presentation and apparent secondary gain. The characteristics of hallucinations and delusions that should trigger consideration of malingering are summarized by Resnick (Table 3) (18). Although these characteristics may be seen in truly psychotic patients, they are unusual enough to provoke further investigation in the inpatient setting.

The most important factor in the suspicion of malingering is the patient's motive and potential for secondary gain. Clinical characteristics, consistency of symptom presentation, extensive interviewing of the patient, and psychometric testing all have their place in the process of evaluating suspected malingerers. But the presence of a strong motive for malingering provides the most reliable starting point for the evaluation. This makes collection of collateral history essential. Information should be sought from as many sources as possible, including law enforcement, relatives, and other mental health professionals familiar with the patient.

\section{EVALUATION OF SUSPECTED MALINGERING PATIENTS}

Suspected malingerers should be interviewed at length and in detail concerning their psychotic symptoms (11). The length of the interview may, in itself, provide useful information. The feigning of psychosis is tiring, and patients with bizarre presentations at the beginning of the interview may appear somewhat bored by the end of it. Open-ended questions should be used, and at first the interviewer should listen as much as possible so that patient can tell their whole story from beginning to

TABLE 2.

\section{Threshold Model for Consideration of Malingering}

Malingering should be suspected when physical or psychiatric symptoms are accompanied by any of the following:

A. Involvement in civil or criminal legal action

B. Potential for combat duty

C. Lack of cooperation with examination and recommendations

D. Complaints greatly in excess of physical findings

E. Apparent environmental incentive for simulation of illness (e.g., obtaining drugs or avoiding work)

F. Suspicion of voluntary control over symptomatology:

1. symptoms worsen when observed, or

2. bizarre or ridiculous symptoms, or

3. symptoms fail to respond to customary treatment

From Cunnien AJ: Psychiatric and Medical Syndromes Associated with Deception, in Clinical Assessment of Malingering and Deception. Edited by Rogers R. New York: The Guilford Press, 1988. 
TABLE 3.

\section{Threshold Model for the Assessment of Hallucinations and Delusions}

Malingering should be suspected if any of the following are observed:

A. Hallucinations

1. Continuous rather than intermittent hallucinations

2. Vague or inaudible hallucinations

3. Hallucinations not associated with delusions

4. Stilted language reported in hallucinations

5. Inability to state strategies to diminish voices

6. Self-report that all command hallucinations were obeyed

B. Delusions

1. Abrupt onset or termination

2. Eagerness to call attention to delusions

3. Conduct not consistent with delusions

4. Bizarre content without disordered thinking

From Resnick PJ: Malingered Psychosis, in Clinical Assessment of Malingering and Deception. Edited by Rogers R. New York: The Guilford Press, 1988.

end. Further questioning should focus on the details of reported symptoms. Patients who are not cooperative with the interview may have to be re-examined repeatedly to obtain the information necessary.

\section{FREQUENT CLINICAL INDICATORS OF DECEPTION INCLUDE $(11,18,19)$ :}

1. Exaggeration or overacting of symptoms. Some malingering patients believe that the more bizarre they appear, the more the clinician will be convinced of their "craziness." In forensic settings, they have been known to act like apes in court, or save bugs and feces to bring to the examination room and eat in front of the examiner. They may report hearing voices "all the time" or state that "everybody is out to get me."

2. Malingerers are eager to call attention to their reported symptoms. When questioned in detail, many will withhold information that is not consistent with "being crazy" and will demonstrate remarkable and convenient gaps in their memory. They tend to repeat questions or answer slowly, to give themselves more time to think of an answer, or may give frequent "I don't know" responses. They may also preface some descriptions with a phrase like, "You may not believe this" or "I know this sounds bizarre." If they feel that the examiner doubts them, they may become angry and ask to see another doctor who "understands their problem." Although the psychomotor retardation found in schizophrenic patients may result in slow and monotonic speech, truly psychotic people are usually reluctant to call attention to their illnesses and rarely emphasize how sick they are or accuse the examiner of not believing their story.

3. Lack of cooperation with the evaluation and treatment process is common in malingering patients. They may become loud and belligerent, attempting to control the interview, and are frequently noncompliant with treatments despite continued claims of psychotic symptoms. An extremely rapid resolution of symptoms after just a few doses of medication is also suspicious, since mood stabilizing and antipsychotic medications require several days before reduction in psychosis is seen. 
4. Malingerers can rarely imitate the form of thought content seen in psychotic patients. Loose or tangential associations, flight of ideas, or consistently odd thought contents are difficult to malinger for any period of time and may help to separate the psychotic patients from their feigning counterparts. Perseveration, frequently an indicator of brain dysfunction, is almost never seen in malingerers.

5. Negative symptoms of schizophrenia, such as blunt affect, social withdrawal, and concrete or idiosyncratic thinking, are almost never seen in malingerers.

6. Symptoms reported by malingering patients usually worsen when they know they are being observed or are being interviewed. Inpatient observation should include consultation with evening and night staff over several days. The majority of the time, malingerers will be revealed to demonstrate behaviors inconsistent with their reports of internal stimuli, paranoia, and bizarre thoughts. It has been noted that mentally ill patients themselves are especially adept at finding malingerers in their midst (14), so reports by fellow patients that "he's faking" should not be dismissed without consideration.

7. Malingerers may report inconsistent symptoms. They may claim not to remember important personal details such as their name or birthdate, which are signs of severe dementia or delirium. Confusing cognitive impairment with psychosis, they may claim not to know the year or the name of the U.S. President despite an apparently clear sensorium. They may also report a hodgepodge of psychiatric symptoms in addition to psychosis including depression, anxiety, racing thoughts, etc.

8. Far-fetched tales of mental illness controlling their behaviors may be reported. The presence of a clear nonpsychotic motive for their presentation, such as escaping arrest or finding shelter, should raise suspicions about the veracity of reported symptoms.

9. The malingerer's current presentation will usually not be consistent with their recent level of psychosocial functioning, as determined from history and collateral contacts. In one recent study (19), this factor was found to have high predictive value for detection of malingering in a forensic setting.

\section{PSYCHOMETRIC TESTING}

Psychometric testing should be performed on suspected malingerers to provide more objective information in favor of or against this diagnosis. The most commonly used psychometric instrument is the revised Minnesota Multiphasic Personality Inventory (MMPI-2). The F-scale, a standard scale of the MMPI-2, has considerable utility in detection of malingered responses (20,21). The raw score of another standard MMPI scale, $\mathrm{K}$, is subtracted from the raw F score to produce the F-K index, which has also been associated with malingered response sets. Hawk et al (21) report that independently identified malingering subjects who completed the MMPI-2 consistently produced high F-scale scores, F-K index scores, and extremely elevated scores on scales describing depression, psychopathic deviancy, paranoia, and schizophrenic characteristics (the so-called "sawtooth" pattern). Use of the MMPI-2 to evaluate suspected malingerers should be done in close cooperation with a clinical psychologist who is familiar with the use of this instrument for this purpose.

To further assist clinicians with the evaluation of suspected malingerers, Rogers 
and colleagues developed the Structured Interview of Reported Symptoms (SIRS). This structured interview, which takes approximately 30 to 45 minutes to administer, has been shown to have considerable efficacy in detecting malingered psychiatric disorders. Validation studies (22) have demonstrated high interrater reliability and ability to discriminate malingered from bona fide psychiatric disorders. It is generally recommended that the SIRS be used in conjunction with a careful clinical interview and the MMPI-2 to provide a more global evaluation.

The $\mathrm{M}$ test was developed by Beaber et al. (23) in an attempt to provide a brief, patient-completed questionnaire for detecting malingered responses. Although initial testing appeared promising, subsequent evaluations indicated that it has limited usefulness in the detection of malingering in clinical populations (24,25). At this time, no brief instrument exists that is useful in emergency settings to screen patients for potentially malingered symptoms.

\section{DIFFERENTIAL DIAGNOSIS OF MALINGERING}

Although research in malingering has emphasized detecting false symptoms based on common presentations, it must be remembered that unusual symptoms do exist. It is also true that mentally ill patients, such as those with schizophrenia, can also exaggerate or malinger symptoms for the same secondary gains that nonmentally ill patients do. Any clinician contemplating a diagnosis of malingering must proceed carefully, always keeping in mind that the major distinguishing factor of malingering is its conscious motivation and potential for secondary gain.

Conversion disorders may be very difficult to differentiate from malingering, since in both disorders objective evidence does not account for reported or observed symptoms. Mutism and catatonia, in particular, may appear as conversion symptoms. Some factors that can assist in differentiation between conversion disorder and malingering are (12):

- Malingerers are usually more aloof and uncooperative, while patients with conversion symptoms tend to be friendly and cooperative.

- Malingerers tend to avoid diagnostic evaluations, while conversion disorder patients welcome them.

- Persons with conversion disorder will generally accept opportunities to get around their disability, while malingerers may resist such efforts.

- Malingerers tend to report detailed descriptions of premorbid events, while conversion disorder patients tend to be vague about historical details.

- While malingerers may express great concern and anxiety about their symptoms, conversion disorder patients classically demonstrate "la belle indifference," an apparent lack of concern about their symptoms.

Schizophrenia and other psychotic disorders must be ruled out, especially in patients with no prior psychiatric history. The time course of the illness, presence or absence of prodromal symptoms, prior psychiatric history, level of psychosocial functioning prior to presentation, and quality of psychotic symptoms should enable the clinician 
to determine if schizophrenia is present. Collateral information is most important in this determination, and should be obtained in all cases.

Delirium should be considered in any patient with acute mental status changes. Most malingerers will not demonstrate the characteristic confusion and cognitive disturbances of delirium prior to their presentation, and metabolic disturbances or toxic ingestions should be relatively simple to exclude by standard laboratory evaluations. The absence of self-neglect (poor grooming, poor hygiene, etc.) would also tend to eliminate delirium from the differential.

Mental Retardation may be considered in patients who appear unable to answer questions appropriately. A history of mental retardation is easily determined by access to school records, prior psychiatric evaluations, and other medical records. Formal intelligence testing should be obtained if any doubt exists. Many malingerers will tend to deliberately miss "easy" questions on IQ testing but will frequently answer "hard" questions correctly (2).

Amnestic disorders may be considered in patients who claim memory gaps. An easily administered screening test, the Rey 15-item test, has been shown to be effective in determining a patient's tendency to exaggerate memory deficits (26). Patients with generalized amnesia still retain basic personal information and usually demonstrate other cognitive deficits in addition to memory loss. Brief, intermittent periods of amnesia are rare in the absence of substance abuse.

Substance abuse, especially alcohol, cocaine, phencyclidine (PCP), and methamphetamine, may create clinical presentations that mimic psychosis. Alcoholic hallucinations are often vivid and frightening, and almost all patients believe they originate outside their head. PCP may cause catatonic mutism. Methamphetamine and cocaine can cause extreme agitation and paranoia with perceptual disturbances. Substance abuse should be confirmed by history and laboratory drug screening. Malingering may be considered if symptoms change or persist following cessation of drug use or detoxification.

Structural brain diseases, especially those involving the frontal lobe, should be ruled out in the case of recent onset psychosis or personality change. The orbitofrontal syndrome, caused by lesions of the inferior caudate nucleus and orbitofrontal cortex, causes impulsiveness, lack of tact, crude behaviors, and lack of concern for the feelings of others. Patients with this disorder could be misdiagnosed with antisocial personality disorder, which demonstrates many of the same characteristics (1) and is associated with malingering (11). Other intracranial pathology may also present with abrupt onset of psychotic symptoms, especially vascular syndromes. Evaluation of these syndromes is best performed using both noncontrasted and contrasted MRI, together with MRA evaluation of the cerebral vasculature.

\section{CONFRONTATION OF MALINGERING PATIENTS}

If the combined impression of extensive interviews, collateral history, psychometric testing, and medical testing indicates malingering, the question arises as to the proper course of action. It is important to remember that malingering patients may 
suffer from severe personality disturbances, such as borderline or antisocial personality disorders, that carry a high risk of aggression and poor impulse control. The "unmasking" of the malingerer will also frustrate their attempt to obtain the intended secondary gain, and they will certainly be angry and embarrassed. The rage unleashed by frustrated malingerers has resulted in numerous assaults on physicians and staff. In Australia, a patient accused of malingering back pain killed two orthopedic surgeons and wounded a third before killing himself. (Autopsy results on the patient indicated no back pathology.) It is clearly in the patient's best interests, as well as the staff's, that confrontation with testing results be done in a way that allows the patient to "save face." In light of the strong negative feelings that the clinician will almost certainly experience when learning that their patient is dishonest, this may not be an easy task.

Opinions given by expert witnesses are considered to be in the service of the court and are protected by testimonial immunity. However, opinions of malingering given by treating physicians are not, and leave the doctor open to potential legal action for misdiagnosis. The physician should have firm, reliable evidence to back up their claim of malingering, all of which must be included in the patient's chart. If the patient is to be discharged from inpatient care, the patient should be told directly by the treating physician. A nonconfrontational style should be adopted throughout the interview. It is better to say, "The evidence that we have does not support a diagnosis of schizophrenia" rather than, "You've been lying to us, and now you're out the door." If the patient suffers from a substance abuse disorder, personality disorder, or other psychiatric condition, they should be referred for outpatient treatment for that particular disorder.

An angry reaction by the patient is expected, and given their motivation, understandable. The clinician should react as they would to any angry, threatening patient, with calm but firm limit setting and measures for the safety of the patient and staff as needed. Verbal threats made to the doctor may occur, and in some instances may be of sufficient severity to warrant notification of law enforcement. However, the physician should not let their strong negative feelings toward the patient color their decision-making, and should not over-react to the malingerer's threats.

\section{CONCLUSION}

Malingering is a difficult diagnosis in psychiatry, a deliberate misuse of the doctor-patient relationship that goes against the instincts of most physicians. A "diagnosis" of malingering requires careful attention to motivation to feign illness, symptom characteristics, historical information, psychometric testing data, and behavioral observations. Due to the pejorative nature of such a diagnosis, the physician must feel confident in their diagnosis before acting on it. It must also be remembered that malingering does not always rule out the presence of mental illness or psychosocial dysfunction. As physicians, we must strive to provide the best psychiatric care available to those in need, while remaining cost-conscious so that the 
maximum number of patients may benefit. The process of evaluating cases of suspected psychiatric malingering, while perhaps distasteful, may provide more access to care for the mentally ill by reducing deliberate misuse of already overburdened resources.

\section{REFERENCES}

1. American Psychiatric Association. The Diagnostic and Statistical Manual of Mental Disorders, 4th Ed. American Psychiatric Association Press, 1994.

2. Resnick PJ: The Detection of Malingered Mental Illness. Beh Sci Law 1984; 2(1): 21-37.

3. Goodwin DW, Alderson P, Rosenthal R: Clinical Significance of Hallucinations in Psychiatric Disorders. Arch Gen Psychiat 1971; 24: 76-80.

4. Assad G, Shapiro B: Hallucinations: Theoretical and Clinical Overview. Am J Psychiatry 1986; 143(9): 1088-1097.

5. Nayani TH, David AS: The auditory hallucination: a phenomenological survey. Psychological Medicine 1996; 26: 177-89.

6. Oulis PG, Mavreas VG, Mamounas JM, Stefanis CN: Clinical characteristics of auditory hallucinations. Acta Psychiatr Scand 1995; 92: 97-102.

7. Falloon IRH, Talbot RE: Persistent auditory hallucinations: coping mechanisms and implications for management. Psychological Medicine 1981; 11: 329-339.

8. Leudar I, Thomas P, McNally D, Glinski A: What voices can do with words: pragmatics of verbal hallucinations. Psychological Medicine 1997; 27: 885-898.

9. Chesterman LP, Boast N: Multi-Modal Hallucinations. Psychopathology 1994; 27: 273 280.

10. Rupert SL, Hollender MH, Mehrhof EG: Olfactory hallucinations. Arch Gen Psychiatry 1961; 5: 121-126.

11. Jaffe ME, Sharma K: Malingering Uncommon Psychiatric Symptoms Among Defendants Charged Under California's "Three Strikes and You're Out” Law. J Forensic Sci 1998; 43(3): 549-555.

12. Mills MJ, Lipian MS: Malingering, in Comprehensive Textbook of Psychiatry, 6th Edition. Edited by Kaplan HI, Saddock BJ. Baltimore: Williams and Wilkins, 1995.

13. Hopkins A, Clarke C: Pretended paralysis requiring artificial ventilation. Br Med J 1987; 294: 961-962.

14. Rosenhan DL: On Being Sane in Insane Places. Science 1973; 179: 250-258.

15. Witztum E, Grinshpoon A, Margolin J, Kron S: The Erroneous Diagnosis of Malingering in a Military Setting. Military Medicine 1996; 161(4): 225-229.

16. Yates BD, Nordquist CR, Schultz-Ross RA: Feigned Psychiatric Symptoms in the Emergency Room. Psychiatric Services 1996; 47(9): 998-1000.

17. Cunnien AJ: Psychiatric and Medical Syndromes Associated with Deception, in Clinical Assessment of Malingering and Deception. Edited by Rogers R. New York: The Guilford Press, 1988.

18. Resnick PJ: Malingered Psychosis, in Clinical Assessment of Malingering and Deception. Edited by Rogers R. New York: The Guilford Press, 1988.

19. Kucharski LT, Ryan W, Vogt J, Goodloe E: Clinical Symptom Presentation in Suspected Malingerers: An Empirical Investigation. J Am Acad Psychiatry Law 1998; 26(4): 579-585.

20. Grow R, McVaugh W, Eno TD: Faking and the MMPI.J Clin Psych 1980; 56(4): 910-917.

21. Hawk GL, Cornell DG: MMPI Profiles of Malingerers Diagnosed in Pretrial Forensic Evaluations. J Clin Psych 1989; 45(4): 673-678. 
22. Rogers R, Kropp PR, Bagby RM, Dickens SE: Faking Specific Disorders: A Study of the Structured Interview of Reported Symptoms (SIRS). J Clin Psych 1992; 48(2): 643-648.

23. Beaber RJ, Marston A, Michelli J, Mills MJ: A Brief Test for Measuring Malingering in Schizophrenic Individuals. Am J Psychiatry 1985; 142: 1478-1481.

24. Hankins GC, Barnard GW, Robbins L: The validity of the $\mathrm{M}$ test in a residential forensic facility. Bull Am Acad Psy Law 1993; 21(1): 111-21.

25. Smith GP, Borum R, Schinks JA: Rule-Out and Rule-In scales for the M test for malingering. Bull Am Acad Psy Law 1993; 21(1): 107-1 10.

26. Leng NRC, Parkin AJ: The Detection of Exaggerated or Simulated Memory Disorder by Neuropsychological Methods. J Psychosomatic Res 1985; 39(6): 767-776. 